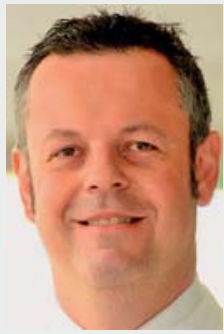

Prof. Dr. med.

Rainer Kollmar

\section{Autoimmunenzephalitis: häufiger als erwartet und behandelbar!}

Enzephalitiden sind häufig schwerwiegende Erkrankungen mit einer hohen Morbidität und Mortalität. In aller Regel denkt der behandelnde Arzt zunächst an eine infektiöse Genese der Erkrankung. Aktuelle Daten zeigen jedoch, dass in bis zu $50 \%$ der Fälle gar keine ursächliche Infektion wie durch neurotrophe Viren festgestellt werden kann. Im California Encephalitis Project konnte in $50 \%$ kein Erreger festgestellt werden [1].

In den letzten Jahren zeigte sich, dass ein beträchtlicher Teil dieser nicht-infektiösen Hirnerkrankungen zu den sogenannten „Autoimmunenzephalitiden (AE)“ gezählt werden kann. Interessanterweise sind die AE häufiger als Enzephalitiden durch Herpes-Simplex-Virus, Varicella-Zoster-Virus und das West-Nile-Virus zusammen [2]. AE werden häufig und lange auf Intensivstationen behandelt und stellen somit eine spezielle Herausforderung für das Intensivteam dar. Die schwerwiegendsten Probleme sind dabei qualitative und quantitative Bewusstseinsstörungen mit Delir, Psychose, Katatonie, mutistischem Stupor bis hin zum Koma, massivste Bewegungsstörungen der Extremitäten (Hyperkinesen) und epileptische Anfälle. Oft kommt es auch zu ausgeprägten autonomen Dysfunktionen (therapierefraktärer Hyperthermie, Herzrhythmusstörungen, Kreislaufdysregulationen). Aufgrund der oft monatelangen Behandlungen im Intensivsetting und der immunsuppressiven Therapie sind Infektionen bis hin zur Sepsis keine Seltenheit. Für das Team selbst kommt es häufig zu kritischen Situationen durch die Dauer der Behandlung, aber auch durch Diskussionen mit der Familie bzgl. Therapieabbrüchen und Limitierungen.

In der aktuellen Ausgabe von Intensivmedizin up2date geben Günther et al. einen sehr differenzierten Überblick über die verschiedenen klinischen Erscheinungsbilder, Diagnosekriterien und Therapiemöglichkeiten der AE [3]. Dieser Artikel ist für jeden Intensivmediziner ein Muss, da das Erkennen der Erkrankung und ihre Behandlungsstrategie ganz entscheidend für das Outcome sind. Selbst schwerste und langwierige Verläufe, insbesondere bei autoimmunen Enzephalitiden, können in einem guten funktionellen Outcome resultieren. Kognitive Langzeitfolgen können sich auch nach Jahren noch bessern. AE zeigen sich klinisch durch fokale neurologische Defizite, kognitive Einschränkungen und/oder epileptische Anfälle. Eine Diagnose ist möglich über Liquordiagnostik, typische EEG-Veränderungen als Hinweis für Enzephalitis und eine auffällige Bildgebung. Nach der Lektüre des Artikels von Günther et al. werden Sie die limbische Enzephalitis, die akute disseminierte Enzephalomyelitis (ADEM), die Anti-NMDA-Rezeptor-Antikörper-Enzephalitis (NMDARE) und die Bickerstaff-Hirnstammenzephalitis erkennen können. Laboruntersuchungen spielen in der Diagnostik eine wichtige Rolle. Die Diagnose ist gesichert, wenn in Liquor und/oder Serum entsprechende Antikörper nachgewiesen werden. Allerdings zeigt sich auch, dass bei 30 bis $40 \%$ der Enzephalitiden, die sehr an eine AE denken lassen, keine Antikörper festgestellt werden können [2,4]. 
In der Akutbehandlung der AE werden Steroidpuls, intravenöse Immunglobuline (IVIG) (off label) und Aphereseverfahren empfohlen, wobei randomisierte Studien fehlen. Die Prognose einer tumorassoziierten Autoimmunenzephalitis ist umso besser, je früher der Tumor behandelt bzw. entfernt wird. Darum sollte eine ausführliche Tumorsuche unbedingt stattfinden. Unter der Akutbehandlung kommt es bei guten Ansprechraten häufig zu einer schnellen klinischen Besserung. Sekundäre multimodale Therapien beinhalten beispielsweise orale Langzeitsteroidgabe, Zytostatika (z. B. Cyclophosphamid) und/oder monoklonale Antikörper (z. B. Rituximab).

Vor Kurzem wurde im Rahmen der „Korea Autoimmune Synaptic and Paraneoplastic Encephalitis Registry (KASPER)“ von $n=80$ Patienten mit AE berichtet, die nach initialer Gabe von Steroiden, IVIGs oder Plasmapherese mit Rituximab behandelt wurden [4]. Die Behandlung der Kontrollgruppe fand ohne Rituximab statt. Interessanterweise wurden 40 \% der Patienten ohne einen positiven Antikörperbefund behandelt. Die Studie zeigte, dass die zusätzliche Behandlung mit Rituximab zu einem deutlich verbesserten Outcome führte - und zwar unabhängig vom Antikörperstatus. Eine frühere Diagnosestellung und frühere Behandlung waren dabei mit einem besseren Outcome assoziiert.

\section{Literatur}

[1] Bale JF Jr. Virus and immune-mediated encephalitides: epidemiology, diagnosis, treatment, and prevention. Pediatr Neurol 2015; 53:3-12

[2] Dubey D, Sawhney A, Greenberg B et al. The spectrum of autoimmune encephalopathies. J Neuroimmunol 2015; 287: $93-97$

[3] Günther A, Schubert ], Witte OW et al. Intensivmedizinische Komplikationen autoimmuner Enzephalitiden. Intensivmedizin up2date 2017; 13: 91-106

[4] Lee W], Lee ST, Byun JI et al. Rituximab treatment for autoimmune limbic encephalitis in an institutional cohort. Neurology 2016; 86: 1683-1691 